\title{
CORONAGRAPH OBSERVATIONS OF THE CORONAL CONDENSATION OF 4 FEBRUARY 1962
}

\author{
H. ZIRIN \\ Hale Observatories, Carnegie Institution of Washington, \\ California Institute of Technology, Pasadena, Calif., U.S.A.
}

(Received 16 October, 1969)

\begin{abstract}
Climax coronagraph observations of the accessible Fe lines, as well as the Caxv $\lambda 5694$ line at the time of the 1962 total eclipse, are analyzed. The spectra show that the ionization equilibrium of iron is not substantially changed in an intense coronal condensation, at least for the stages $\mathrm{x}$ through Xv. The only exception is Fexv $\lambda 7059$, for which density effects are important. The stability of the ionization distribution is explained by the dynamic nature of the Fe ionization, with ions entering on the high side (Fexvi and up) due to rapid heating and then cooling through the visible stages.

Comparison of the ionization distributions inferred from radiative and collisional excitation of the iron lines shows that the excitation must be by collisions everywhere at the heights examined (less than $50000 \mathrm{~km}$ ).

The iron abundance in the corona is found to be $10^{-4}$ that of hydrogen, but this figure would be reduced by the amount of cyclic excitation.

The peak electron density in the condensation is $8 \times 10^{9}$, and the peak value of the 25694 line/ continuum ratio is 2.5 , in good agreement with calculations by Chevalier and Lambert.

The ratio of the infrared FexmI lines is measured along the limb and found to vary with electron density as expected, the $10747 / 10798$ ratio is 7 or less at densities much below $10^{9}$ and saturates at a value of 2 for densities above that amount.
\end{abstract}

\section{Introduction}

Shortly before the total solar eclipse of 4 February $1962 *$, coronal spectrograms of a series of iron lines were obtained by Robert James, following the Climax "iron series" program (Firor and Zirin, 1962). The lines observed are Fex 6374, Fe XI 7892, FexIII 10747 and 10798 , Fexiv 5303, Fexv 7059, and Caxv 5694. Some of the data for 5694 and 10747 have already been reported (Zirin, 1964; Malville, 1967).

Table I lists the spectrograms obtained and Figure 1 shows the spectrograms at a single height. Because the telescope had to be used for routine coronal surveys at the exact eclipse time, our spectra are not exactly comparable with those made at the eclipse site, but comparison with the routine survey shows no great change in the corona in this two hour period. A complete set of spectra was also obtained February 5 , but there is no significant difference from those of February 4.

The spectra were traced on the Baird microdensitometer of the High Altitude Observatory by Louise Mao and Helen Glasco and on the Sinclair Smith microdensitometer at Caltech by the author. Each line was traced at a number of position angles and heights. Calibration is relative to the center of the sun in the usual way. We

* This eclipse is often referred to as occurring on February 5, 1962, which was the local date; however the time of most of the eclipse observations in New Guinea was 2250 UT, February 4, 1962.

Solar Physics 11 (1970) 497-512. Copyright () 1970 by D. Reidel Publishing Company, Dordrecht-Holland. All Rights Reserved. 
TABLE I

List of Climax spectra 4-5 February 1962

\begin{tabular}{llll}
\hline Climax No. & Time & $\lambda$ & Height interval $(\mathrm{km})$ \\
\hline Feb. 4, 1962 & & & \\
2920 & $1947-2037$ & $\begin{array}{l}10747,10798 \\
(+10830)\end{array}$ & 9000 \\
2921 & $2102-2109$ & 7892 & 4500 \\
2922 & $2117-2140$ & $7060(+7065)$ & 4500 \\
2923 & $2216-2227$ & $6374(+6563)$ & 4500 \\
2924 & $2201-2208$ & $5303+5694$ & 4500 \\
2925 & $2234-2256$ & $6374+6363$ & 4500 (complete limb survey) \\
2926 & $2301-2313$ & 5303 & \\
& & & \\
Feb. 5, 1962 & & $7060+7065$ & 4500 \\
2927 & $2214-2225$ & 7892 & 4500 \\
2928 & $2201-2209$ & 10747,10798, & 9000 \\
2929 & $2011-2108$ & +10830 & 4500 \\
2930 & & $5303+5694$ & 4500 \\
2931 & $2257-2305$ & $6374+6563$ & \\
\hline
\end{tabular}

determined line widths where possible. When line widths could not be measured accurately they were estimated from the values for other lines. Peak intensities are calibrated to the center of the disk by spectra made there with a triangular slit and filters of known attenuation. The absolute values are probably good to $\pm 10 \%$ for the stronger lines (with the exception of 7892, as noted below. The product of the intensity and line width are used to estimate the integrated intensity, considering the line as a Gaussian. Table II summarizes these data for a number of points on the limb for all lines. For each position angle, data are given for height intervals of $4500 \mathrm{~km}$ (the zero is not well established, but Ht. 1 is probably around $9000 \mathrm{~km}$ above the photosphere). At each entry, four quantities are given: $\lambda I$ (in every case $I$ implies the integrated line intensity) in units $10^{4} \mathrm{erg} / \mathrm{ster} / \mathrm{cm}^{2} / \mathrm{sec}$ and $\lambda I / g_{U}\left(g_{U}\right.$ is the statistical weight of the upper level producing the line) in the upper row; and $I g_{L} / I S \lambda$ in units $10^{-6} /$ micron $\left(g_{L}\right.$ is the statistical weight of the lower level and $S$, the line strength) and $\Delta \lambda / \lambda \times 10^{4}$ in the lower row. The first quantity, used by Firor and Zirin (1962), is proportional to the total number of photons in the line, with no questions asked; the second is proportional to the number of ions if excitation is by collision; the third gives the same for photoexcitation; and the fourth is the normalized width at halfintensity. For three lines, 7060, 7892, and 10830 an instrumental width of about $\frac{1}{2} \AA / \mathrm{mm}$ has been subtracted (by Pythagorean difference) but of course the integrated intensity is proportional to the measured half-width.

Because of the low dispersion and inferior quality of the infrared spectra, only the line widths for 6374 and 5303 are reliable. These show two important points, already indicated by previous work:

1. The Doppler widths of a given line tend to be constant from point to point, regardless of coronal temperature changes. 

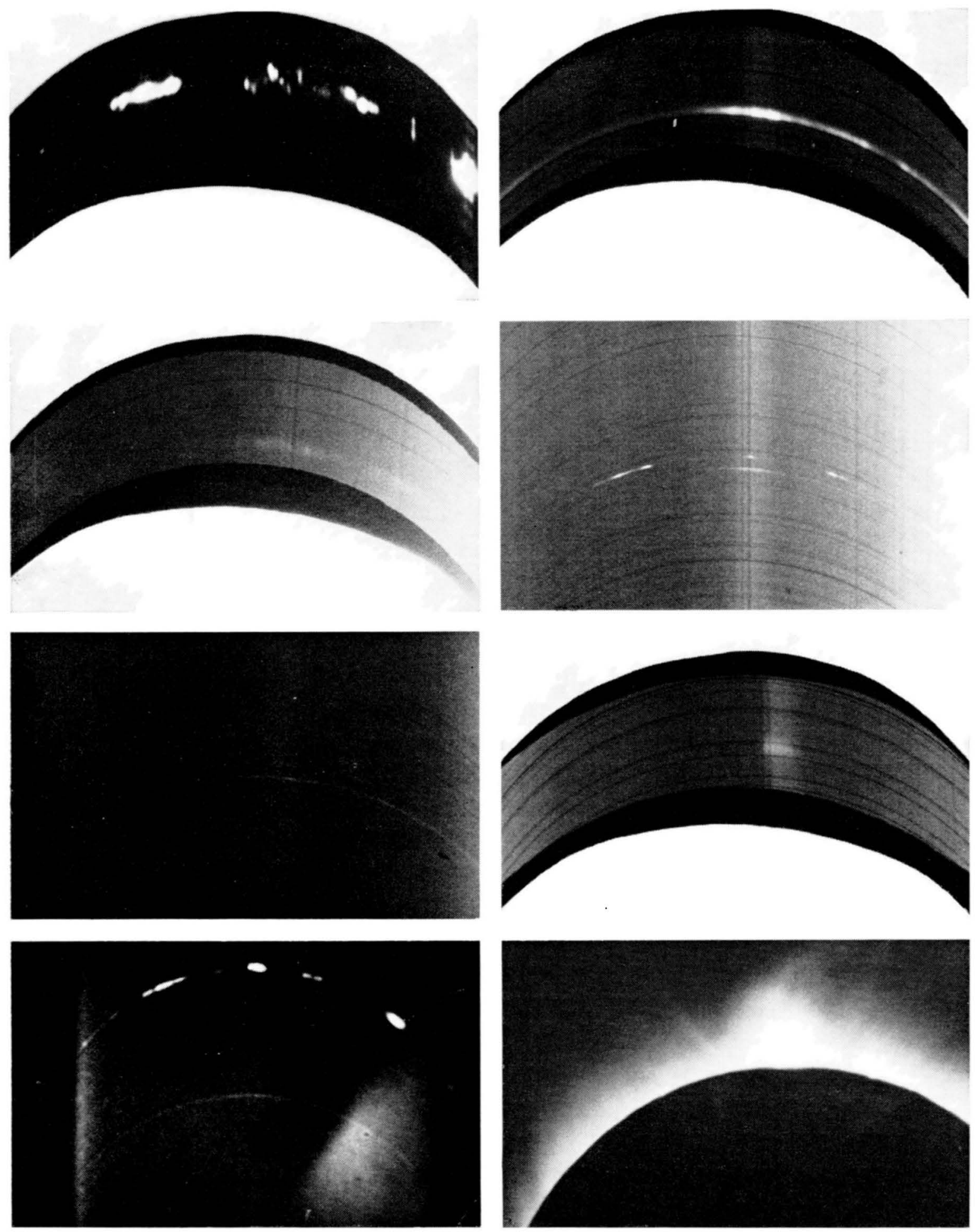

Fig. 1. Coronal lines photographed with the Climax coronagraph simultaneously with the February 4, 1962, eclipse. Shown are: (a) H $\alpha$; (b) Fex, $6374 \AA$; (c) FexI, 7892; (d) HeI (10 830), Fe XIII (10 798 and 10 747); (e) FexIv, 5303; (f) HeI (7065), (g) Caxv, 6594; (h) a white-light photograph printed on the same scale. 
2. The thermal broadening for Fex is always less than that for FexIv; the average ratio is 0.86 , suggesting a temperature ratio of 0.73 .

\section{The Ionization Equilibrium of Iron}

The implications of the foregoing facts for the understanding of the physics of the corona are often overlooked. First, it is clear that the observed widths of each line trace out the temperature at which that line is most favorably emitted, and can give us no information on the dominant coronal temperature at any point. Second, the temperature difference between lines shows that there is considerable temperature fine structure; the fact that this fine structure is seen in condensations shows that it is local, rather than a line of sight superposition of different regions. We must remember that deduced ionization distributions always apply to a mixture of temperatures.

In view of the existence of temperature fine structure we can consider two alternatives: either there is a fixed temperature gradient between hotter and colder regions, or all coronal material passes through a sequence of temperatures. Since the ionization-recombination time is of the order of seconds, ionization equilibrium is always established. There alternatives might be sorted out from the observed ionization distributions: In active regions, the first would lead to an increase in the relative number of higher ionization stages because the relative function of higher temperature regions would be increased. In the second model less change would be observed; there would be increased input of hot gas to the corona, but this would still flow through all the temperature stages. A broadening of the ionization distribution toward higher ionization stages would result.

In order to infer the distribution of $\mathrm{Fe}$ ions in different stages of ionization from the observed intensities, we must determine whether photoionization or collisions are dominant. We attempt to do this by comparing ionization curves derived for each circumstance.

The formula for photoexcitation given by Firor and Zirin (1962) is:

$$
I=N_{i} L F_{U L} \frac{h}{4 \pi}=\frac{1}{8 \pi} \frac{g_{2}}{g_{1}} \frac{A_{U L} h}{e^{h v / k T}-1} N_{i} L
$$

where $N_{i}$ is the number of ions $/ \mathrm{cm}^{3}$, and $L$, the length of the condensation. Since we measure the ratio of this intensity to that per unit angstrom of photosphere, we have:

$$
\begin{aligned}
\frac{I}{I} & =\frac{1}{8 \pi} \frac{g_{2}}{g_{1}} \frac{A_{U L} h}{e^{h v / k T}-1} \frac{\lambda^{5}}{2 h c^{2}}\left(e^{h v / k T}-1\right) \times 10^{8} N_{i} L \\
& =\frac{10^{8}}{16 \pi} \frac{g_{u}}{g_{L}} \frac{\lambda^{4}}{c} A_{U L} N_{i} L .
\end{aligned}
$$

The factor $10^{8}$ is from our use of the photospheric intensity per angstrom. Since

$$
A_{U L}=\frac{35300}{g_{U}} \frac{912^{3}}{\lambda(\AA)} S
$$


TABLE II

Coronal line intensities at different points

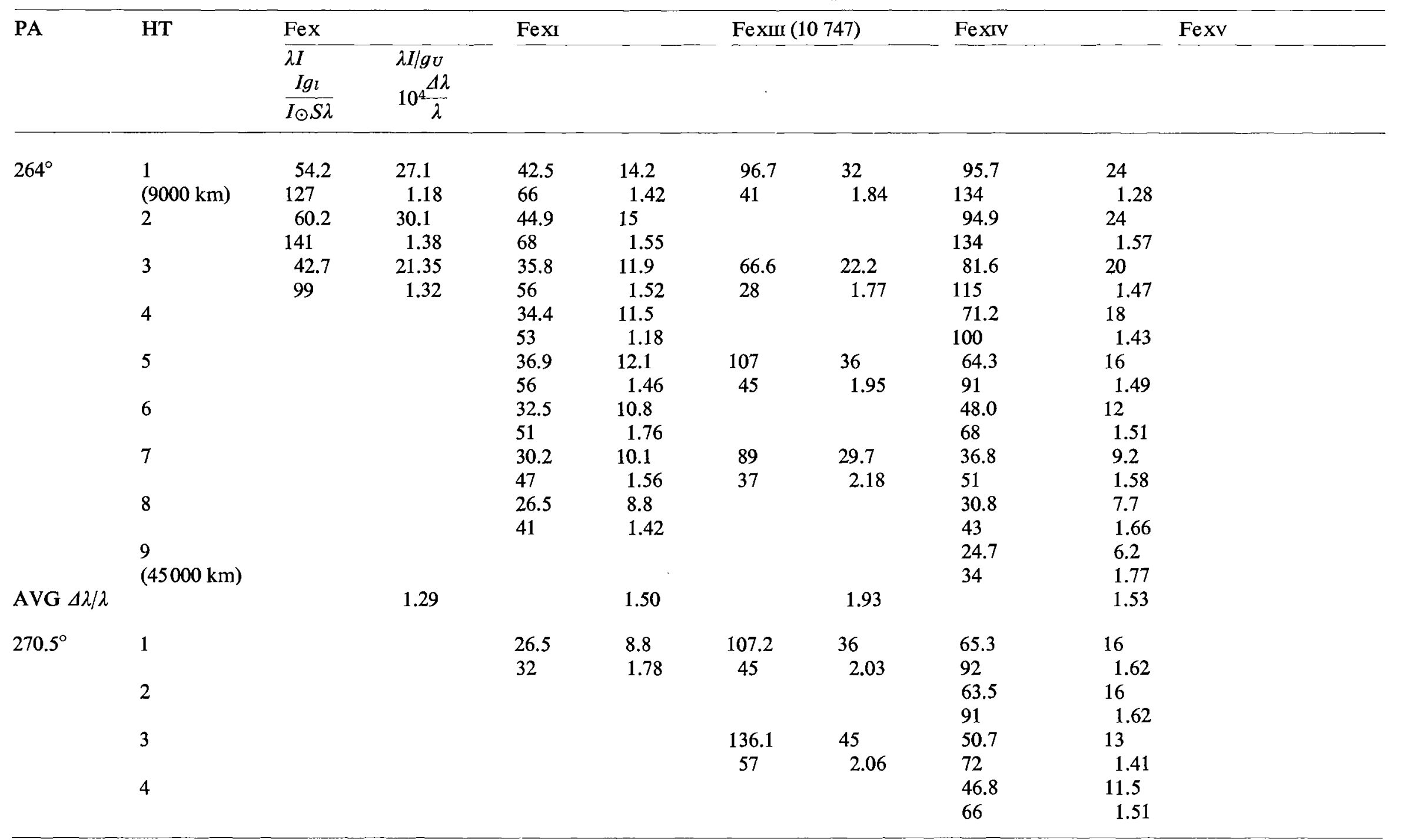


Table II (continued)

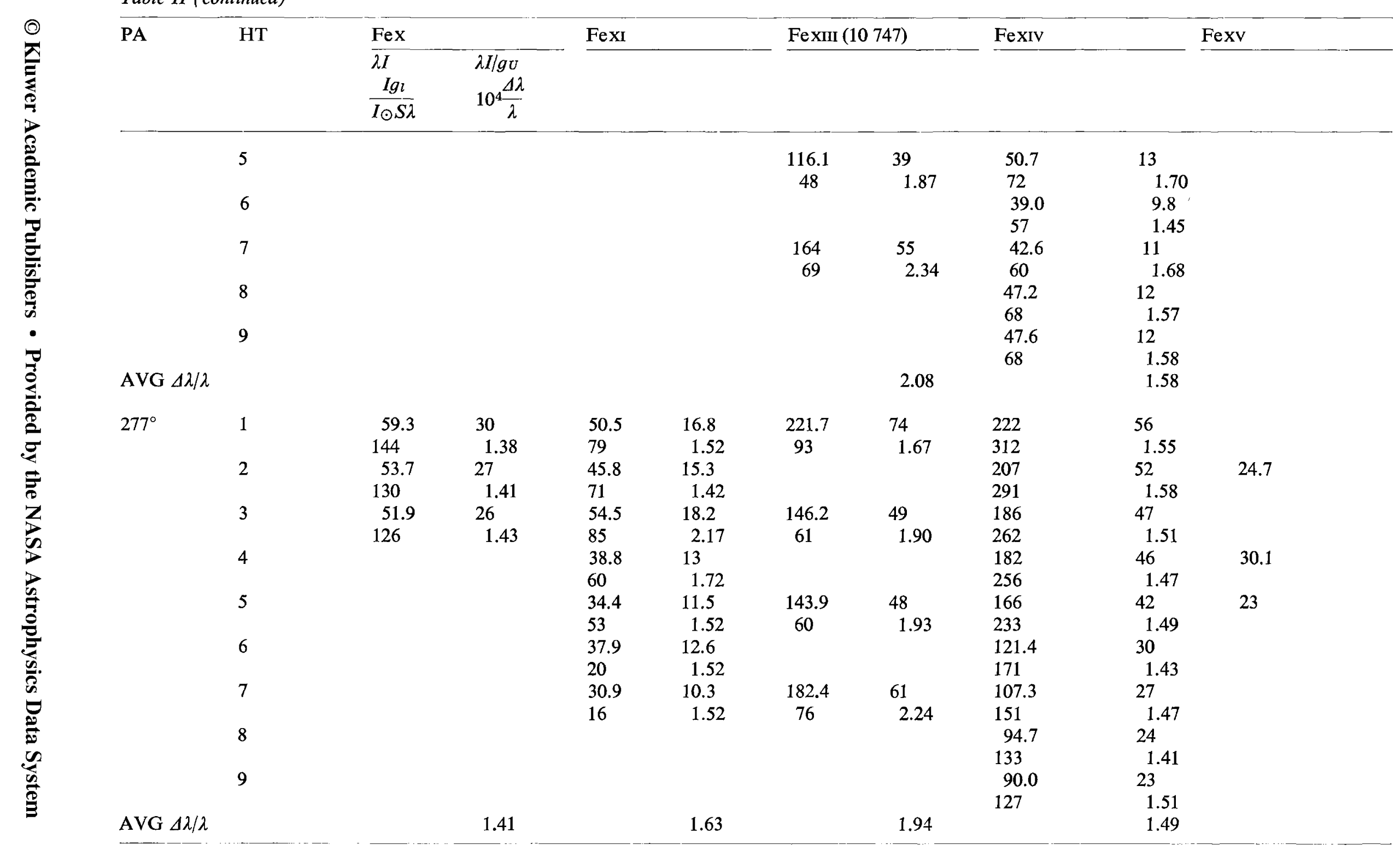




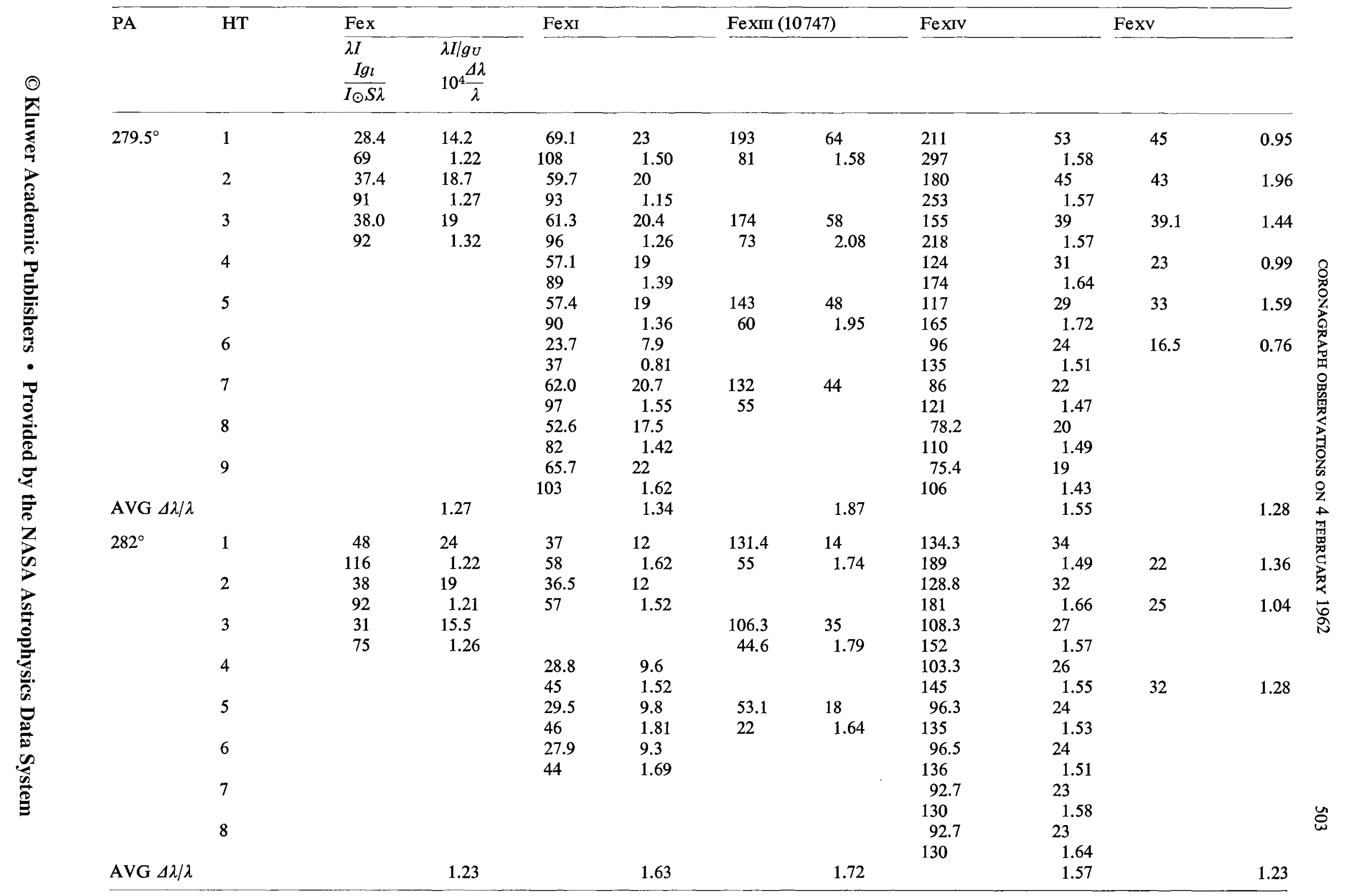


Table II (continued)

\begin{tabular}{|c|c|c|c|c|c|c|c|c|c|c|}
\hline \multirow[t]{2}{*}{ PA } & \multirow[t]{2}{*}{ HT } & \multicolumn{2}{|l|}{ Fex } & \multicolumn{2}{|l|}{ FexI } & \multicolumn{2}{|c|}{ FexIII (10747) } & \multirow[t]{2}{*}{ Fexiv } & & \multirow[t]{2}{*}{ Fexv } \\
\hline & & $\begin{array}{l}\overline{\lambda I} \\
\frac{I g_{l}}{I \odot S \lambda}\end{array}$ & $\begin{array}{l}\lambda I / g_{U} \\
10^{4} \frac{\Delta \lambda}{\lambda}\end{array}$ & & & & & & & \\
\hline AVG $\Delta \lambda / \lambda$ & $\begin{array}{l}1 \\
2 \\
3 \\
4 \\
5 \\
6 \\
7 \\
8 \\
9\end{array}$ & $\begin{array}{l}14.7 \\
36 \\
16.6 \\
40\end{array}$ & $\begin{array}{l}7.3 \\
1.33 \\
8.3 \\
1.46\end{array}$ & $\begin{array}{l}30.9 \\
48 \\
35.2 \\
55\end{array}$ & $\begin{array}{c}10.3 \\
1.52 \\
11.7 \\
1.50\end{array}$ & $\begin{array}{l}94 \\
39 \\
\\
69.2 \\
29\end{array}$ & $\begin{array}{l}31 \\
2.27 \\
23 \\
2.22\end{array}$ & $\begin{array}{l}30.8 \\
43 \\
29.1 \\
41 \\
24.9 \\
35 \\
23.7 \\
33 \\
24.5 \\
34 \\
17.5 \\
25 \\
15.5 \\
5.5 \\
14.5 \\
5.1 \\
12.9 \\
4.5\end{array}$ & $\begin{array}{l}7.7 \\
1.47 \\
7.3 \\
1.66 \\
6.2 \\
1.66 \\
5.9 \\
1.51 \\
6.1 \\
1.87 \\
4.4 \\
1.40 \\
3.9 \\
1.43 \\
3.6 \\
1.51 \\
3.2 \\
1.28 \\
1.53\end{array}$ & $<2$ \\
\hline
\end{tabular}


where

$$
S=\frac{\left[J^{2}-(L-S)^{2}\right]\left[(L+S+1)^{2}-J^{2}\right]}{4 J}
$$

we have

$$
\frac{I}{I_{\odot}}=\frac{10^{-16}}{16 \pi} \frac{1}{g_{L} c} \frac{\lambda}{c} 35,300(912)^{3} S N_{i} L .
$$

Thus to derive the ionization distribution for the quiet corona, we should multiply the derived values of $I / I_{\odot}$ by $g_{L} / S \lambda$. These are given in the lower left of each quartet of numbers in Table II.

If the density is sufficiently high for collisional excitation to play a role, or if the line in question (e.g. 7059 or 10798 ) can only be excited by collisions with either electrons or protons, the intensity of a line would be approximately

$$
\frac{I}{I_{\odot}}=\frac{C_{12} h v N_{i} L}{I_{\odot}}
$$

where $C_{12}$ will depend irregularly on atomic parameters, exhibiting only a proportionality to $g_{U}$. Thus to evaluate the ionization distribution under higher density conditions we should multiply the intensity measure $I / I_{\odot}$ by $I_{\odot} / g_{U}$, and by $1 / C_{12}$ if reliable values are available. Since the tabulated values of $C_{12}$ do not differ much from one another, and a sizable (and not completely determined) correction for cyclic excitation has to be made, we have omitted $C_{12}$ and simply given $\lambda I / g_{U}$ in Table II, assuming that any collisional process will be proportional to $g_{U}$. The various hypotheses for excitation are then checked by plotting the resultant ionization distributions for each position angle, using an average of two or three of the lowest graded heights. These plots, shown in Figure 2, strongly suggest that the excitation is by collisions. There is no reason to expect the dips in the ionization distributions obtained in the radiative excitation case, particularly if we average over a range of temperatures. If the excitation in the condensation is indeed by collisions, then the similarity of the ionization pattern inside and outside of the condensation suggests that excitation is everywhere by collisions. It is interesting that the plot of $\lambda I$, the "no questions asked" method originally used by Firor and Zirin (1962), shows the smoothest ionization distribution. This would be expected if cyclic excitation via higher levels is important; the great variety of excitation paths would tend to even out the various excitation cross-sections. Since known collisional excitation cross-sections are inadequate for simple collisions to dominate at typical coronal densities, we conclude that cyclic excitation is important.

The curves of ionization show a minimum at FexI in a number of cases. On the other hand, the raw intensity of the 7892 line is high; it shows measurable intensity at a range of position angle and intensity exceeded only by 5303. Thus, the absolute calibration is suspect, and we must assume that the dip at FexI is an artifact of our calibration procedure. Unfortunately, the filters used are not available for recalibration. 


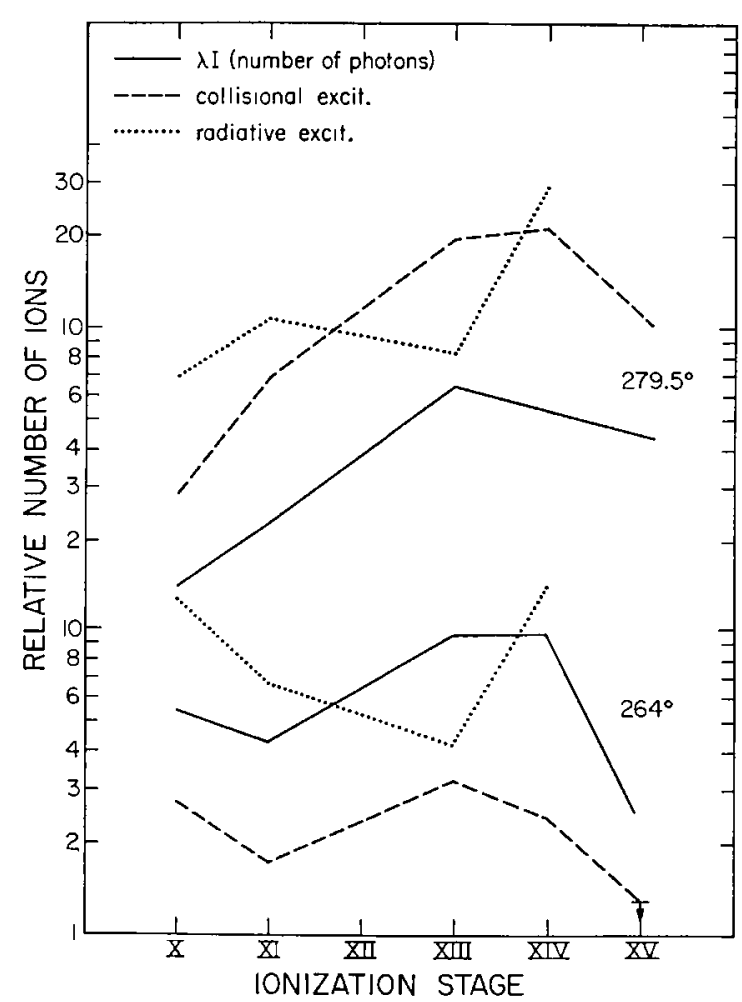

Fig. 2. Relative number of ions of different ionization stages of three different assumptions for two position angles.

We see that the derived ionization distributions clearly correspond to excitation by collisions. To check this result we use collateral criteria which do not depend on our guess for the appropriate ionization equilibrium.

\section{Criteria for the Importance of Collisional Excitation}

From Equation (5) we can derive observational criteria $g_{U}$ for the onset of collisional excitation. First we can calculate the line/continuum ratio. The scattered light intensity from coronal electrons is (Zirin, 1964)

$$
\frac{I_{c}}{I_{\odot}}=1.6 \times 10^{-25} N_{e} L
$$

so that the line to continuum ratio with radiative excitation is

$$
\frac{I_{L}}{I_{c}}=\frac{1.75 \times 10^{-15}}{1.6 \times 10^{-25}} \cdot \frac{\lambda}{g_{L}} \frac{S N_{i} L}{N_{e} L}=1.1 \times 10^{10} \frac{\lambda}{g} S N_{i} N_{e}
$$

where $N_{i}$ is the number of ions in the given state. For $\lambda 5303 S=\frac{4}{3}, g_{u}=4$, giving

$$
\frac{I_{L}}{I_{c}}=1.94 \times 10^{6} \frac{N_{i}}{N_{e}}
$$

If the $\mathrm{Fe}$ abundance is $10^{-4}$ that of $H$, and $\frac{1}{5}$ of this is FexIv, the line/continuum ratio would be 60 . Unfortunately, the continuum is so weak that it can only be observed 
with the coronagraph in coronal condensations in which the most abundant ions are far above FexIv. In the quiet corona (e.g. P.A. $264^{\circ}$ in our data) the continuum is less than $2 \times 10^{-6} I_{\odot}$, giving $I_{L} / I_{c}>25$. Thus we can get no definite information about the line/continuum ratio from coronagraph observations.

Malville (1967) has shown that the line/continuum ratio for 10747 in the condensation is greater than 50 . Our values, obtained by comparing continuum measured in the visible with the line measured in the infrared, are much lower. I suspect Malville had difficulties measuring the I.R. continuum accurately. In any event, the continuum emission is predominantly from the Caxv region and associated with 4000000 degree material so that the intensity of the continuum to be compared with 10747 is considerably less than the measured value.

We see a key difficulty in absolute abundance analyses from coronagraph spectra. If the continuum is strong enough for a line/continuum ratio measurement, most of the continuum comes from a hot region in which the observable iron ions are probably not very abundant. Furthermore, the abundance rests on understanding the mechanisms of the complex processes of cyclic and direct collisional excitation. On the other hand, if we can find a low density region where the easily understood process of photoexcitation is dominant, the continuum will surely be too weak to be measurable. However, in either case a lower limit for abundance is obtained; in view of the famous overabundance of iron in the corona (or underabundance in the photosphere), that is most important.

Since the continuum is undetectable in quiet regions, it is useful to have a lineintensity criteria for the radiative excitation. This is obtained from Equation (5); using the value $N_{i} / N_{e}=2 \times 10^{-5}$, (consonant with an $\mathrm{Fe} / \mathrm{H}$ ratio of $10^{-4}$ ) for the ion population, we have for $\lambda 5303$

$$
\frac{I_{5303}}{I_{\odot}}=1.2 \times 10^{-24} N_{e} L .
$$

The region of enhanced 5303 on our spectra covers about $20^{\circ}$ of limb, or $2.4 \times 10^{10}$ $\mathrm{cm}$ (the intense condensation covers $2^{\circ}$ ). If we, alternatively, assume the length is determined by the scale height of coronal emission we get a similar number.

$$
\frac{I_{5303}}{I_{\odot}} \sim 2.93 \times 10^{-14} N_{e} .
$$

With $N_{e}=10^{9}$, we see the highest possible $\lambda 5303$ intensity obtainable from photoexcitation alone is $30 \times 10^{-6} I_{\odot}$. Since the observed values of intensity exceed this value, we must have collisional excitation.

Using Blaha's (1968) cross-section we find a collisional excitation rate of $0.784 \times 10^{-9}$ $N_{e}$ at $2 \times 10^{6} \mathrm{deg}$; the photoexcitation rate is $0.66 \mathrm{sec}^{-1}$. For $N_{e} \sim 10^{9}$, collisional excitation just competes with photoexcitations; Equation (11). If we use Chevalier and Lambert's (1969) calculation of cyclic excitation, we get twice as much collisional excitation. This would explain the high coronal intensities (60 millionths) in quiet regions. 
Another criterion was given by the author (Zirin, 1968) in terms of the ratio $R$ of the numbers of photons in the lines $\lambda 5303$ and $\lambda 7059$. When this ratio is large, collisions are unimportant; when $R$ is small, they are significant. The density may also be derived:

$$
N_{e} \sim \frac{2 \times 10^{9}}{R-4} \quad \text { where } \quad R=\frac{\lambda I_{5303}}{\lambda I_{7059}}
$$

( $R$ is never less than 4).

Conversely, using Equation (4) of that paper, the ratio of collisional to radiative excitation is

$$
\frac{F_{12}}{C_{12}}=0.33 R-1 .
$$

So our criterion is:

$$
\frac{F_{12}}{C_{12}}<1 \text { if } \frac{I_{5303}}{I_{7059}}<8 .
$$

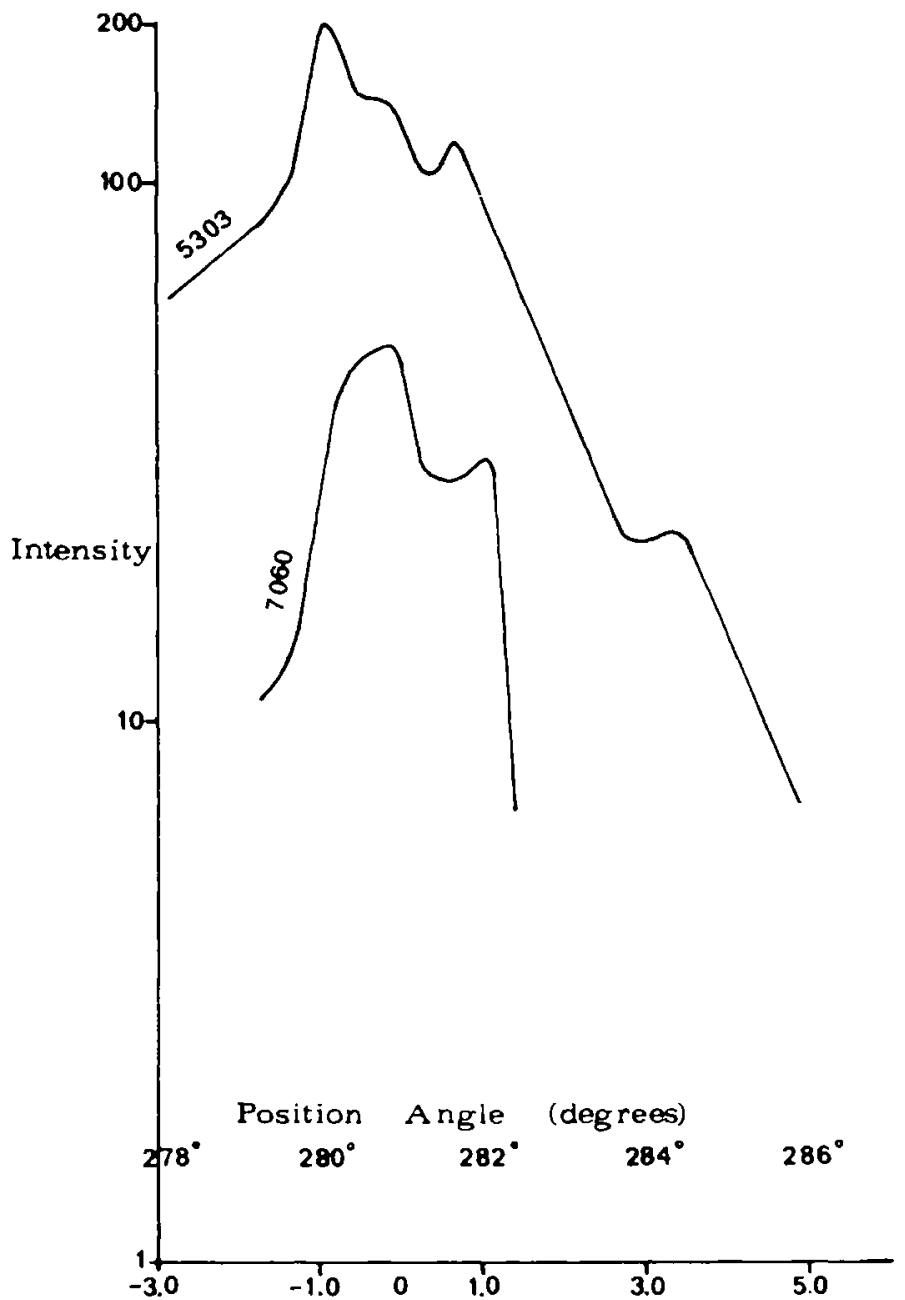

Fig. 3. Intensity distribution of FexIv $\lambda 5303$ and Fexv $\lambda 7060$ in the coronal condensation of February 4, 1962. 
In Figure 3 we show the distribution of the number of photons in 7059 and 5303 (expressed as $\lambda I$ ) in the concentration. The sharp drop in the 7059 intensity outside the condensation suggests that collisional excitation is not important outside at least for 7059; the cross-sections for 7059 excitation are small, and collisional excitation of 5303 will occur at lower densities. Because 7059 is a triplet line, it is probably less affected by cyclic excitation than other coronal lines. It would be useful to obtain such data for another iron line for which cyclic collisions are important but photoexcitation is not.

In Figure 4 we have plotted the distribution of intensity of each Fe coronal line as

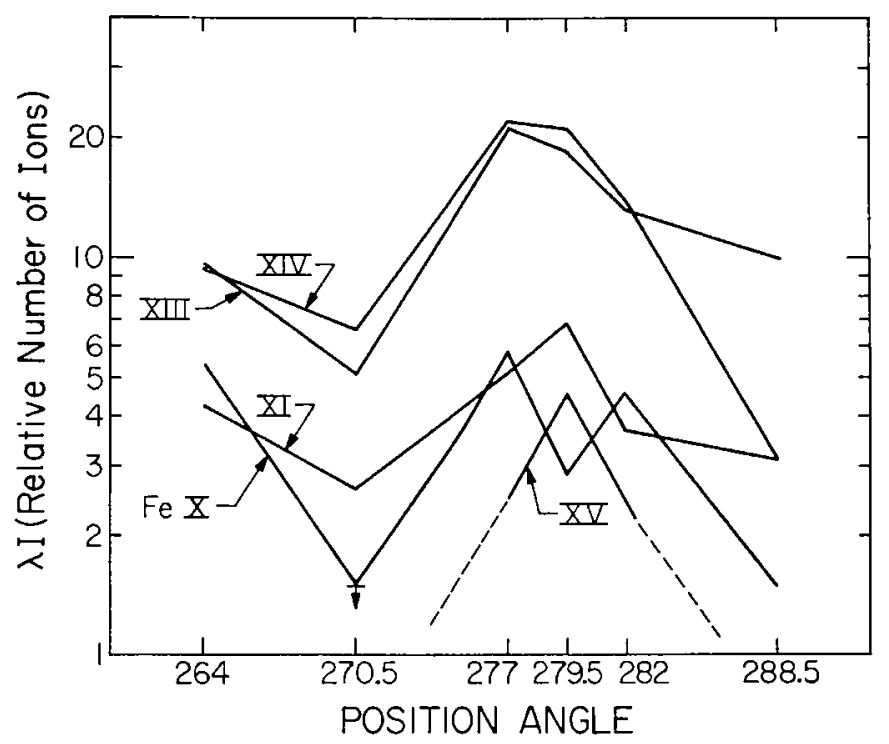

Fig. 4a. Distribution of population in each ion across the corona condensation.

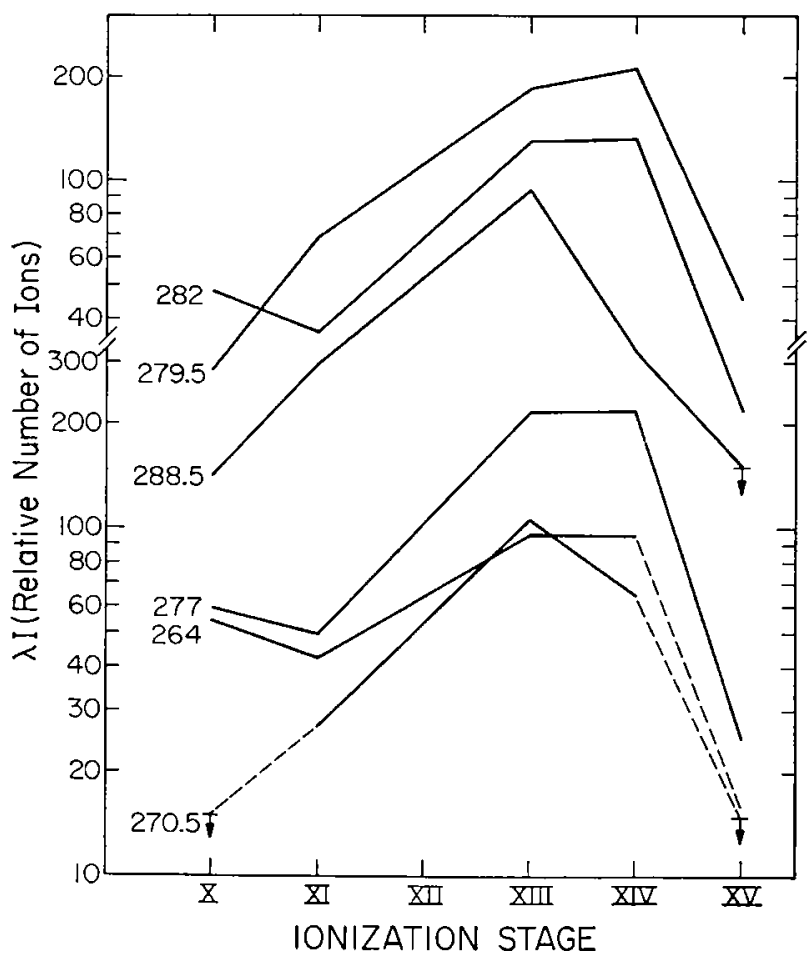

Fig. 4b. Ionization disposition at each position angle. 
a function of position angle. This makes clear the effect of the coronal condensation.

On the basis of all these criteria we conclude that collisional excitation is certainly dominant in the condensation, and since relative line intensities are similar outside the condensation, the same must be true there. This means that the effect of cyclic excitation must be considerable, for ordinary collision rates are inadequate to overbalance photoexcitation. Another possibility is that the corona is highly filamented and the lines are preferentially emitted from denser regions.

In Figure $4 \mathrm{a}$ we have plotted the relative ionization at each point and in Figure $4 \mathrm{~b}$ we give the variation of population at each point, all under the assumption made by Firor and Zirin that the population of each ionic state is proportional to the number of photons observed. The curves are monotonously the same, and give the depressing feeling that there is not much to be learned about variation in density and temperature from plots of this sort (except for the Fexv variation).

\section{The Caxv Lines and the Continuun}

A set of excellent spectra of the yellow coronal line 5694 of Caxv were obtained simultaneously with the 5303 line. Unfortunately, spectra in the second Caxv line (5445) were not obtained. Contours of the continuum intensity outside the line are plotted in Figure 5; the line to continuum ratio at each point is indicated. The con-

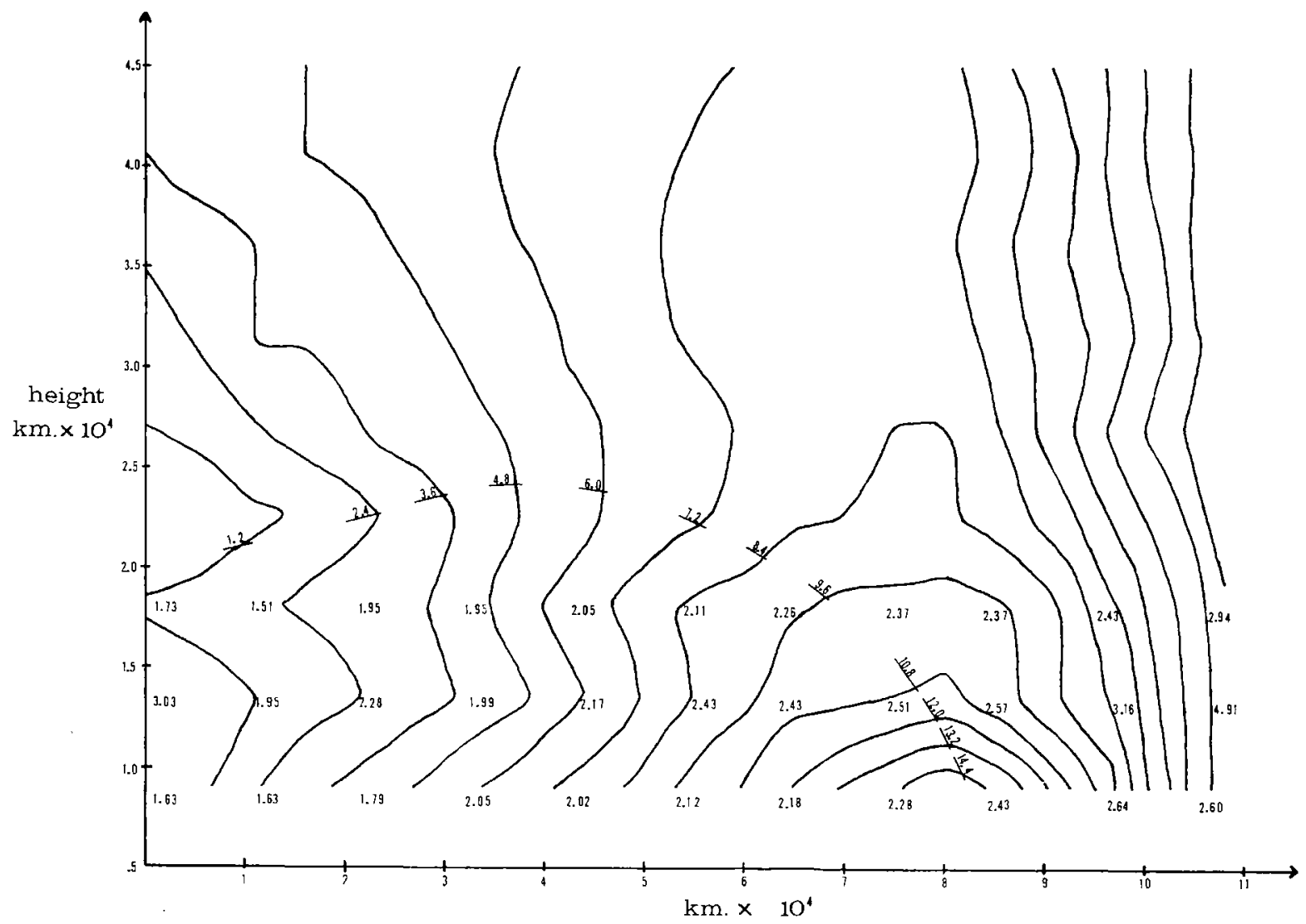

Fig. 5. Contours of continuum emission near $\lambda 5694$. February 4, 1962 condensation. Contour interval: $1.2 \times 10^{-6} I_{\odot}$. Grid numbers give $\lambda 5694$ line/continuum ratio. 
tours were made by tracing normal to the dispersion; the integrated line intensities correspond to the average line width of $1.3 \AA$. It is easy to see the drop in line/continuum ratio as we go to lower densities.

The brightness contours of Figure 5 may be compared with those published by Waldmeier (1965) based on his eclipse measurements. We find a peak value at $H=1.02$ $R$ of $11 \times 10^{-6}$; Waldmeier found $10 \times 10^{-6}$ * Since about 30 minutes elapsed between our yellow line observations and the eclipse some small change might be expected in any event.

There is, of course, an obvious contradiction in this analysis. The continuum data gives the total number of electrons at any temperature; we cannot use the same material to produce both the iron and calcium lines. Presumably, the bulk of the condensation is $4 \times 10^{6}$ degrees, and produces the Caxv lines, because that density is needed for the observed intensity. The abundance of $\mathrm{Fe}$ is so great that the iron lines could all be produced by a fraction of the material at a lower temperature.

\section{The FexIII Infrared Lines}

We have already mentioned the discussion of the 10747 intensities by Malville (1967). Firor and Zirin showed that the relative intensity of 10798 and 10747 should vary with density, because 10798 cannot be excited directly by photoexcitation. More refined calculations by Chevalier and Lambert (1969) take into account proton excitation, which preferably excites the upper state, as well as cyclic processes.

The infrared lines are very difficult to measure because of the low quality of the image tube phosphor and the faintness of 10798 . We have made rough measurements of the intensity of both lines on the Grant measuring engine at Caltech, using the amplitude measured on the oscilloscope as a measure of intensity. The signal to noise ratio is somewhat greater this way than on the microdensitometer. The results for the lowest height at a series of points around the limb are given in Table III along with electron densities derived from the continuum measurements. There is clearly a steep rise in the 10798 intensity in the coronal condensation, where the density

\section{TABLE III}

Relative intensity of the infrared coronal lines

\begin{tabular}{llrlr}
\hline Pos. Ang. & $\lambda 10747$ & $\lambda 10798$ & Ratio & $N_{e}$ (Meas.) \\
\hline 264 & 14 & 2 & 7 & $10^{9}$ \\
270 & 15 & 7 & 2.1 & $10^{9}$ \\
277 & 18 & 10 & 1.8 & $4 \times 10^{9}$ \\
282 & 14 & 6 & 2.3 & $4 \times 10^{9}$ \\
288.5 & 12 & 4 & 3 & $2 \times 10^{9}$ \\
\hline
\end{tabular}

* It is clear from these continuum intensities that the line/continuum ratio for 10747 cannot be 50, for then the 10747 line intensity would exceed 500 millionths of the disk. Thus Malville (1969) must be in error. 
is higher. However, as can be seen from the table, the agreement is only rough, as the line ratio appears to stay at $2: 1$ even at $270 \mathrm{deg}$, where the density is somewhat lower. But there is no doubt that the ratio is quite high in the coronal regions where the density is surely less than $10^{9}$. The line ratio appears to saturate at two for relatively low densities like $10^{9}$, more or less in agreement with the results of Chevalier and Lambert (1969).

\section{Conclusions}

We have carefully examined a complete set of coronal spectra made at the time of the 1962 total eclipse with the Climax coronagraph. The spectra show that the ionization equilibrium of iron is not substantially changed in an intense coronal condensation, at least for the stages $\mathrm{x}$ through $\mathrm{xv}$. The increase in Fexv emission in the condensation may be explained by density effects. This result is explained by the dynamic nature of the Fe ionization, with material being fed in on the high side (FexvI and up) and cooling through the lower stages.

Comparison of ionization distributions inferred from radiative and collisional excitation of the iron lines shows that the excitation must be by collisions everywhere at the heights examined (less than $50000 \mathrm{~km}$ ).

The iron abundance in the corona is found to be $10^{-4}$ that of hydrogen, but this figure would be reduced by the amount of cyclic excitation.

The peak electron density of the condensation is $8 \times 10^{9}$, and the peak value of the $\lambda 5694$ line/continuum ratio is 2.5 , in good agreement with calculations by Chevalier and Lambert.

The ratio of the infrared FexIII lines is measured along the limb and found to vary with electron density as expected; the $10747 / 10798$ ratio is 7 or less at densities much below $10^{9}$ and saturates at a value of 2 for densities above that amount.

\section{Acknowledgements}

I am indebted to the late Bob James for obtaining the spectra, to Helen Glasco and Louise Mao for assisting with the measurements, and to David Lambert and Roger Chevalier for use of the unpublished calculations, as well as many discussions. This work was begun at the High Altitude Observatory and concluded at Caltech under sponsorship of NASA Grant NGR 05-002-034.

\section{References}

Chevalier, R. and Lambert, D. L.: 1969, Solar Phys. 10, 115.

Firor, J. W. and Zirin, H.: 1962, Astrophys. J. 135, 122.

Malville, J. M.: 1967, Astrophys. J. 148, 229.

Waldmeier, M.: 1965, Astrophys. J. 61, 186.

Zirin, H.: 1964, Astrophys. J. 140, 1216.

Zirin, H.: 1968, Astrophys. J. 151, 383. 